\title{
The evaluation of the role of medial collateral ligament maintaining knee stability by a finite element analysis
}

Dong Ren ${ }^{1,2}$, Yueju Liu' ${ }^{1,2}$, Xianchao Zhang ${ }^{1,2}$, Zhaohui Song ${ }^{1,2}$, Jian Lu² ${ }^{1,2}$ and Pengcheng Wang ${ }^{1,2,3^{*}}$

\begin{abstract}
Background: A three-dimensional finite element model (FEM) of the knee joint was established to analyze the biomechanical functions of the superficial and deep medial collateral ligaments (MCLs) of knee joints and to investigate the treatment of the knee medial collateral ligament injury.

Methods: The right knee joint of a healthy male volunteer was subjected to CT and MRI scans in the extended position. The scanned data were imported into MIMICS, Geomagic, and ANSYS software to establish a threedimensional FEM of the human knee joint. The anterior-posterior translation, valgus-varus rotation, and internalexternal rotation of knee joints were simulated to observe tibial displacement or valgus angle. In addition, the magnitude and distribution of valgus stress in the superficial and deep layers of the intact $M C L$ as well as the superficial, deep, and overall deficiencies of the MCL were investigated.
\end{abstract}

Results: In the extended position, the superficial medial collateral ligament (SMCL) would withstand maximum stresses of $48.63,16.08,17.23$, and $16.08 \mathrm{MPa}$ in resisting the valgus of knee joints, tibial forward displacement, internal rotation, and external rotation, respectively. Meanwhile, the maximum stress tolerated by the SMCL in various ranges of motion mainly focused on the femoral end point, which was located at the anterior and posterior parts of the femur in resisting valgus motion and external rotation, respectively. However, the deep medial collateral ligament could tolerate only minimum stress, which was mainly focused at the femoral start and end points.

Conclusions: This model can effectively analyze the biomechanical functions of the superficial and deep layers of the MCLs of knee joints. The results show that the knee MCL $\|^{\circ}$ injury is the indication of surgical repair.

Keywords: Biomechanics, Finite element, Knee joint, Medial collateral ligament, Model

\section{Background}

The medial collateral ligament (MCL) plays an important role in limiting and maintaining the movement of the knee joint and protecting its stability [1]. There is a high incidence of injury to the knee MCL in sports activities such as ice hockey, skiing, and soccer [2], accounting for approximately $40 \%$ of all severe knee joint injuries, $50 \%$ of which involve partial fracture while $30 \%$ involve complete fracture and injury of the knee MCL [3]. These injuries may ultimately lead to medial

\footnotetext{
* Correspondence: zhengzainingmeng@163.com

${ }^{1}$ Third Hospital of Hebei Medical University, Shijiazhuang 050051, China

${ }^{2}$ Hebei Provincial Key Laboratory of Orthopaedic Biomechanics, Shijiazhuang 050051, Hebei, China

Full list of author information is available at the end of the article
}

laxity and instability of the knee joints, as well as secondary long-term complications. Most surgeons [4] advocate conservative treatment for the knee MCL $\mathrm{I}^{\circ}$ injury and surgical repair for the knee $\mathrm{MCL} \mathrm{III}^{\circ}$ injury, respectively. However, the option to deal with $\mathrm{MCL} \mathrm{II}^{\circ}$ injury is controversial. This study is to evaluate the function in detail within MCL maintaining the stability of the knee joint and expects to provide evidence on how to treat the knee MCL II injury.

\section{Methods \\ General information}

A healthy male volunteer (age, 27 years; height, $177 \mathrm{~cm}$; weight, $75 \mathrm{~kg}$ ) without any right knee deformity, history 
of trauma, or clinically positive signs was selected for the study. He consented to participate in this test by signing an informed consent.

\section{Acquisition of CT and MR imaging data}

The right knee joint of the volunteer was subjected to continuous spiral CT in a relaxation and extended position, from $95 \mathrm{~mm}$ above the upper margin of the patella to $110 \mathrm{~mm}$ below the knee joint line, i.e., from the middle lower segment of the femur to the middle upper segment of the tibiofibula. The scan parameters were as follows: layer thickness of $0.7 \mathrm{~mm}$, matrix size of $512 \times 512$, and pixel size of $0.705 \mathrm{~mm}$; in total, 369 Digital Imaging and Communications in Medicine (DICOM)-format images were acquired.

MR imaging was performed for the same right knee joint in the same position, from $50 \mathrm{~mm}$ above the upper margin of the patella to $70 \mathrm{~mm}$ below the knee joint line, in which the axial T1W1 sequence was selected. The scan parameters were as follows: TR of $1900 \mathrm{~ms}$, TE of $2.58 \mathrm{~ms}$, layer thickness of $1 \mathrm{~mm}$, matrix size of $256 \times 256$, and pixel size of $0.859 \mathrm{~mm}$; a total of 176 DICOM-format images were obtained.

\section{Establishment of bone tissue model of knee joints based on CT images}

The obtained CT data were imported into an interactive medical image control system, Materialise Interactive Medical Image Control System (MIMICS) 14.0 (Materialise, Leuven, Belgium). A three-dimensional model of the original bone tissue of the knee joint was obtained using the threshold segmentation and threedimensional model calculation and was imported into automatic reverse engineering software, Geomagic Studio 12.0 (Geomagic, USA), for optimization, so as to obtain a finer bone tissue model. The model was again imported into MIMICS 14.0 software, which was initially meshed in the 3-matic module, and the 4-node tetrahedral element was transformed into a 10-node tetrahedral element.

\section{Establishment of ligament and meniscus models based on MR images}

The method was basically the same as mentioned above, except for the following aspects: (1) Due to the unclear boundary between the soft tissues in the MIMICS 14.0 workspace, individual planes of the meniscus and ligaments were required to be split manually, followed by calculation to obtain the original meniscus and ligament models of the knee joints. (2) In some MCLs, differentiating the superficial and deep layers was difficult; they required to be separated using the trimmer, stretching, Boolean subtraction, and other functions in Geomagic Studio 12.0 according to their length, width [5], thickness ratio, and differences in their other normal anatomic structures, by obtaining their fine models. (3) Before ligament and meniscus models were initially meshed in the 3-matic module of MIMICS 14.0 software, they were subjected to Boolean subtraction calculation in Geomagic Studio 12.0 software to obtain the threedimensional models.

\section{Finite element partition and analysis Model assembly}

Bone tissues, ligaments, and meniscus models were saved in cdb format and imported into the workbench of ANSYS 13.0 software (ANSYS, USA). The models were then assembled, and material properties were applied as per the properties reported in the literature $[6,7]$ (Table 1). Contact of the starting and ending points of each ligament with the bones, and that of the superficial and deep layers of the medial collateral ligament with the meniscus were defined as bonded contact, while contacts at other sites were defined as "no separation contacts." The models were remeshed using an interactive mesh of pentahedral and hexahedral elements, and a total of 877,070 nodes and 354,003 elements were obtained, as shown in Fig. 1.

\section{Loads and boundaries}

The upper femur was fixed in 6 degrees of freedom (DOF), and 134-N forward force, 134-N backward force, $10-\mathrm{N} \mathrm{m}$ valgus torque, and $10-\mathrm{N} \mathrm{m}$ external rotation torque and internal rotation torque were applied to the femur.

Table 1 Material parameters of the normal finite element model

\begin{tabular}{lll}
\hline Structure & $E(\mathrm{MPa})$ & $V$ \\
\hline Femur & 3883.4 & 0.3 \\
Tibia & 4184.6 & 0.3 \\
Fibula & - & 0.3 \\
Patella & - & 0.3 \\
Menisci & 59 & 0.3 \\
ACL & 1.046 & 0.4 \\
PCL & 1.035 & 0.4 \\
SMCL & 1.063 & 0.4 \\
DMCL & 1.063 & 0.4 \\
LCL & 1.063 & 0.4 \\
PL & 1.035 & 0.4 \\
\hline
\end{tabular}

$A C L$ anterior cruciate ligament, $D M C L$ deep medial collateral ligament, $E$ Young's modulus, $L C L$ lateral collateral ligament, $P C L$ posterior cruciate ligament, $P L$ patellar ligament, $S M C L$ superficial medial collateral ligament, $\checkmark$ Poisson's ratio 


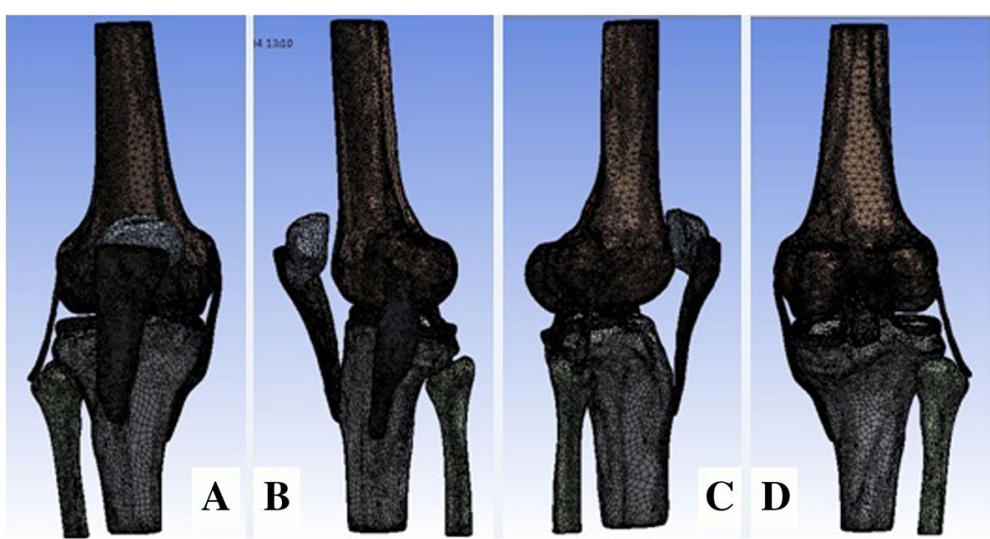

Fig. 1 Mesh generation in ANSYS Workbench. a Anterior view. b Medial view. c Lateral view. d Posterior view

\section{Calculation and post processing}

The tibial displacement or valgus angle as well as the stress magnitude and distribution in the superficial and deep layers of medial collateral ligaments under conditions of intact MCL (case 1) as well as superficial MCL (SMCL) deficiency (case 2), deep MCL (DMCL) deficiency (case 3), and overall deficiencies of the MCL (case 4) is described in Fig. 2.

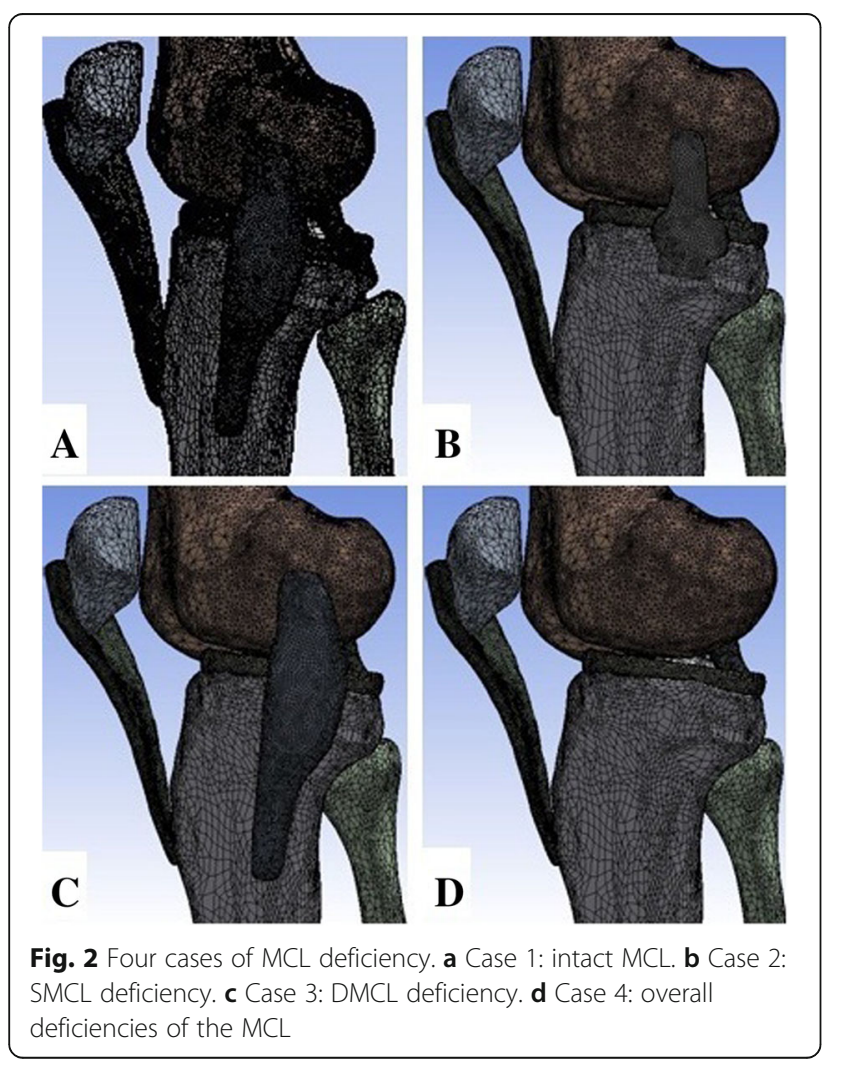

\section{Model validation}

The tibial anterior translation was observed to be $4.89 \mathrm{~mm}$ when constraining the 6 DOF in the upper femur and applying a forward force of $134 \mathrm{~N}$ to the tibia in an extension position of the knee joint; the translation was reported to be $4.6-5.0 \mathrm{~mm}$ using the same load in previous studies [8]. Thus, our results were consistent with the previously reported results on FEM studies, suggesting the effectiveness of our model.

\section{Results}

Under the load of the 134- $\mathrm{N}$ forward force, the tibial displacement changed from $4.89 \mathrm{~mm}$ at intact MCL to 5.17, 5.04, and $5.17 \mathrm{~mm}$ at SMCL deficiency, DMCL deficiency, and overall MCL deficiency, respectively. The peak stress was maximum at the anterior cruciate ligament (ACL), lower at SMCL, and minimum at DMCL (Table 2) and was mainly located at the femoral end point at both ACL and SMCL and at the start and end points at DMCL (Fig. 3).

Under the load of the 134-N backward force, the tibial displacement changed from $4.98 \mathrm{~mm}$ at intact MCL to 4.99, 4.92, and $5.02 \mathrm{~mm}$ at SMCL deficiency, DMCL deficiency, and overall MCL deficiency, respectively. The

Table 2 Response parameters of the knee joint under a force of $134 \mathrm{~N}$ in anterior translation

\begin{tabular}{llllll}
\hline & $\begin{array}{l}\text { Tibial } \\
\text { displacement }(\mathrm{mm})\end{array}$ & \multicolumn{5}{l}{ Peak stress (MPa) } \\
\cline { 3 - 6 } & & $\mathrm{ACL}$ & $\mathrm{PCL}$ & $\mathrm{SMCL}$ & $\mathrm{DMCL}$ \\
\hline Case 1 & 4.89 & 23.31 & 14.70 & 15.18 & 7.95 \\
Case 2 & 5.17 & 26.60 & 14.40 & - & 8.73 \\
Case 3 & 5.04 & 24.84 & 14.83 & 16.08 & - \\
Case 4 & 5.27 & 27.38 & 14.83 & - & - \\
\hline
\end{tabular}

$A C L$ anterior cruciate ligament, $D M C L$ deep medial collateral ligament, $P C L$ posterior cruciate ligament, $S M C L$ superficial medial collateral ligament 

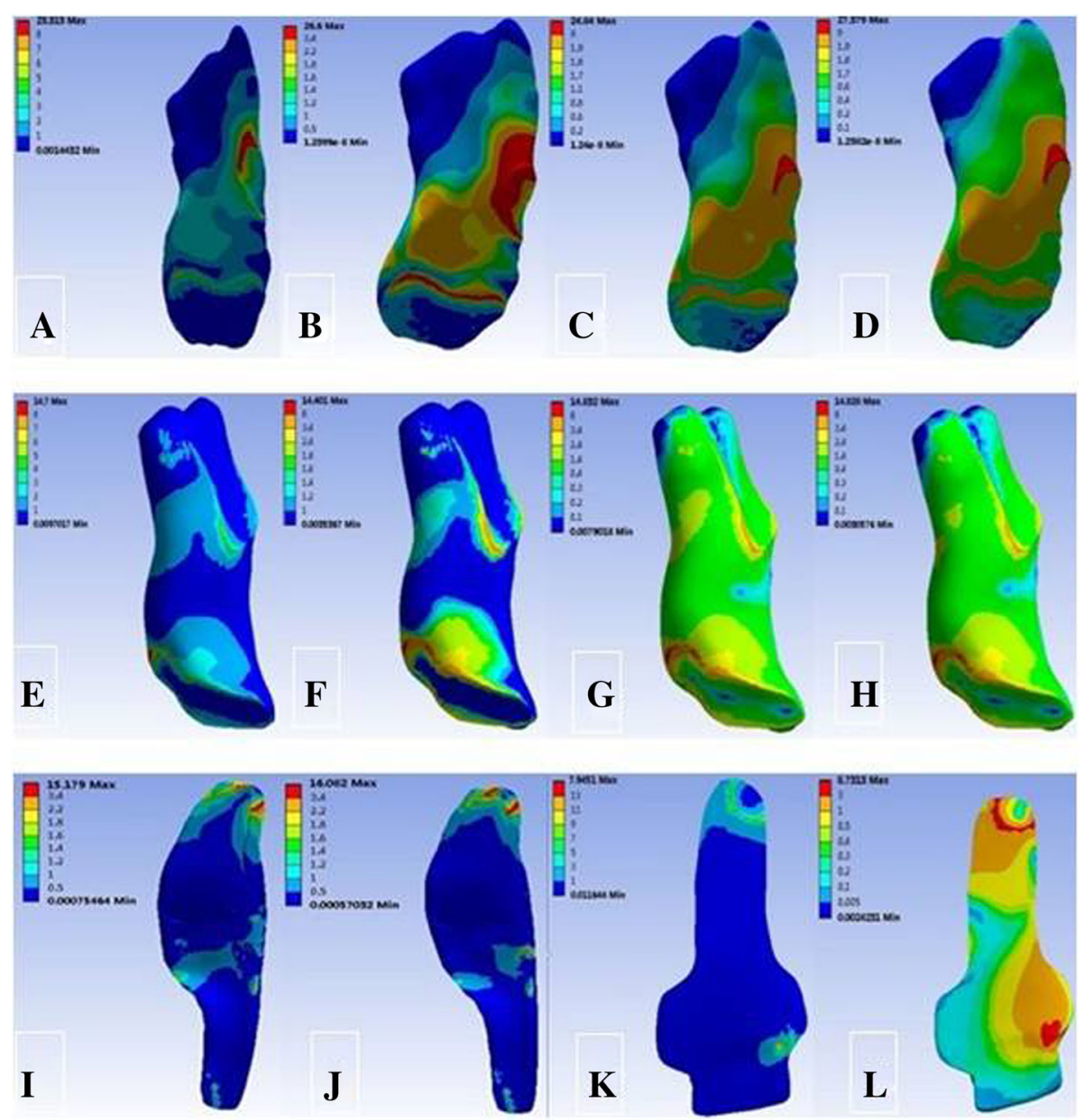

Fig. 3 von Mises stress distribution of the $A C L, P C L, S M C L$, and DMCL under a force of $134 \mathrm{~N}$ in anterior translation. a $A C L$ in case 1. b $A C L$ in case 2. $\mathbf{c ~ A C L}$ in case 3. $\mathbf{d} \mathrm{ACL}$ in case 4. e PCL in case 1. $\mathbf{f} \mathrm{PCL}$ in case 2. $\mathbf{g} \mathrm{PCL}$ in case 3. $\mathbf{h} \mathrm{PCL}$ in case 4. i SMCL in case 1. $\mathbf{j} S M C L$ in case 3. $\mathbf{k}$ DMCL in case 1. I DMCL in case 2

peak stress was maximum at the posterior cruciate ligament (PCL), lower at SMCL, and very low at DMCL (Table 3) and was mainly located at the femoral start and end points at PCL and DMCL and only at the femoral end point at SMCL (Fig. 4).

Under the load of the $10-\mathrm{N} \mathrm{m}$ valgus torque, the tibial valgus angle changed from $4.06^{\circ}$ at intact MCL to $6.08^{\circ}$,

Table 3 Response parameters of the knee joint under a force of $134 \mathrm{~N}$ in posterior translation

\begin{tabular}{llllll}
\hline & \multirow{2}{*}{$\begin{array}{l}\text { Tibial } \\
\text { displacement }(\mathrm{mm})\end{array}$} & \multicolumn{5}{l}{ Peak stress (MPa) } \\
\cline { 3 - 6 } & & $\mathrm{ACL}$ & $\mathrm{PCL}$ & $\mathrm{SMCL}$ & $\mathrm{DMCL}$ \\
\hline Case 1 & 4.98 & 10.68 & 26.32 & 7.26 & 3.44 \\
Case 2 & 4.99 & 12.62 & 26.32 & - & 6.78 \\
Case 3 & 4.92 & 11.90 & 26.32 & 8.40 & - \\
Case 4 & 5.02 & 12.72 & 26.32 & - & - \\
\hline
\end{tabular}

$A C L$ anterior cruciate ligament, $D M C L$ deep medial collateral ligament, $P C L$ posterior cruciate ligament, $S M C L$ superficial medial collateral ligament $4.86^{\circ}$, and $6.22^{\circ}$ at SMCL deficiency, MCL deficiency and overall MCL deficiency, respectively. The peak stress was maximum at SMCL and gradually decreased at DMCL, ACL, and PCL (Table 4); it was mainly located at the femoral end point and anterior part at SMCL and at the femoral start and end points at DMCL (Fig. 5).

Under the load of the $10-\mathrm{N} m$ external rotation torque, the tibial external rotation angle changed from $5.92^{\circ}$ at intact $\mathrm{MCL}$ to $5.95^{\circ}, 5.94^{\circ}$, and $6.10^{\circ}$ at SMCL deficiency, DMCL deficiency, and overall MCL deficiency, respectively. The peak stress was maximum at SMCL, relatively lower at ACL and PCL, and the lowest at DMCL (Table 5). The peak stress at SMCL was mainly located at the end point and posterior part of the femur, and that at DMCL was located at the start and end points (Fig. 6).

Under the load of the $10-\mathrm{N} \mathrm{m}$ internal rotation torque, the tibial internal rotation angle changed from $6.64^{\circ}$ at intact MCL to $7.48^{\circ}, 6.72^{\circ}$, and $7.57^{\circ}$ at SMCL deficiency, 

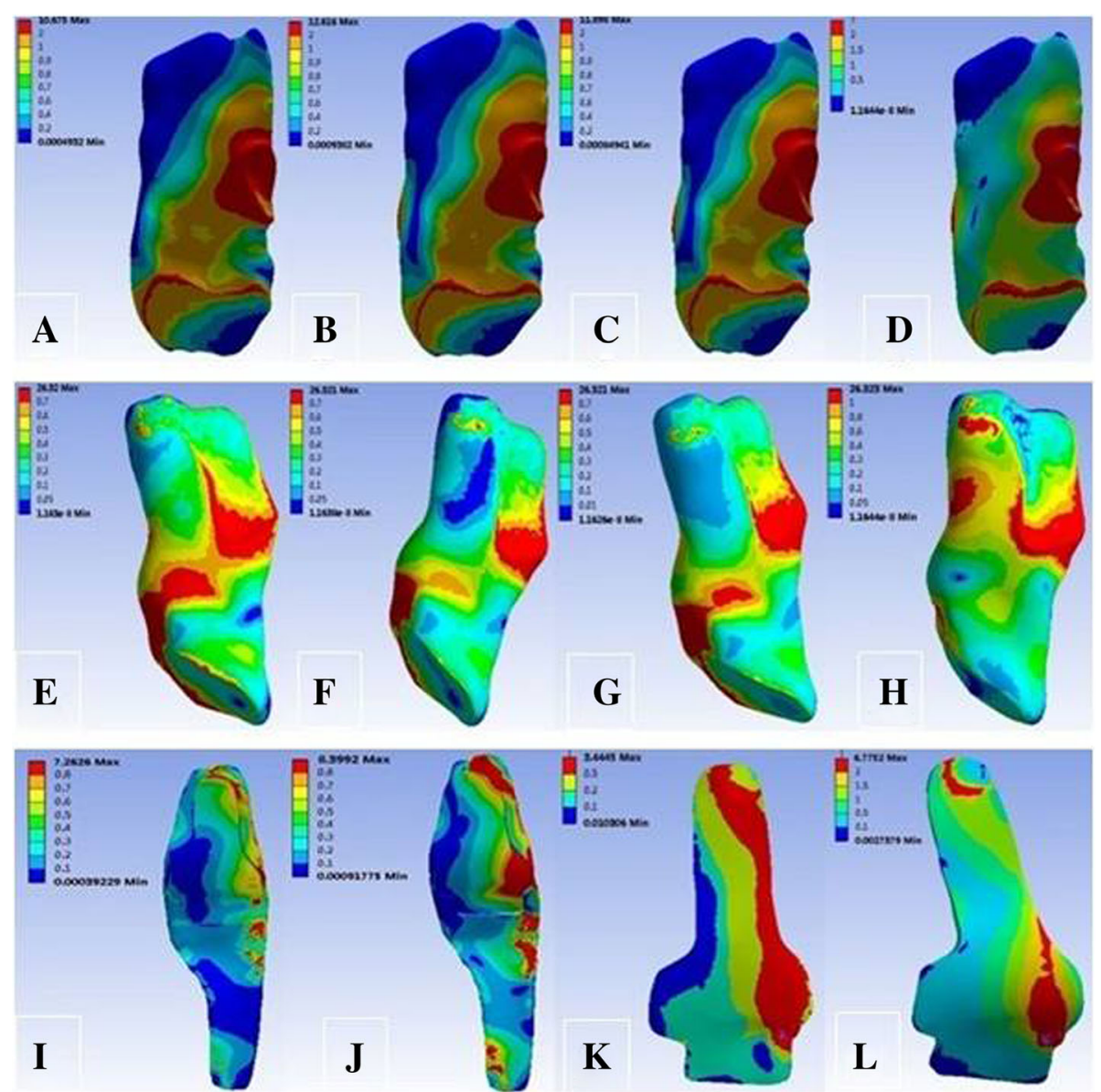

Fig. 4 von Mises stress distribution of $A C L, P C L, S M C L$, and $D M C L$ under a force of $134 \mathrm{~N}$ in posterior translation. a $A C L$ in case 1 . $\mathbf{b} A C L$ in case 2 . c $A C L$ in case 3. $\mathbf{d} A C L$ in case 4. e PCL in case 1. $\mathbf{f} P C L$ in case 2. $\mathbf{g} P C L$ in case 3. $\mathbf{h} P C L$ in case 4. $\mathbf{i} S M C L$ in case 1. $\mathbf{j}$ SMCL in case 3. $\mathbf{k} D M C L$ in case 1. I DMCL in case 2

DMCL deficiency, and overall MCL deficiency, respectively. Meanwhile, the peak stress was maximum at SMCL, relatively lower at ACL and PCL, and minimum at DMCL (Table 6) and was mainly located at the femoral end point at SMCL and at the femoral start and end points at DMCL (Fig. 7).

Table 4 Response parameters of the knee joint under $10 \mathrm{~N} \mathrm{~m}$ of valgus moment

\begin{tabular}{llllll}
\hline & $\begin{array}{l}\text { Tibial valgus } \\
\text { angle }\left(^{\circ}\right)\end{array}$ & \multicolumn{4}{l}{ Peak stress $(\mathrm{MPa})$} \\
\cline { 3 - 6 } & & $\mathrm{ACL}$ & $\mathrm{PCL}$ & $\mathrm{SMCL}$ & $\mathrm{DMCL}$ \\
\hline Case 1 & 4.06 & 6.77 & 4.88 & 30.17 & 9.49 \\
Case 2 & 6.08 & 12.01 & 10.14 & - & 16.11 \\
Case 3 & 4.86 & 9.40 & 8.40 & 48.63 & - \\
Case 4 & 6.22 & 20.22 & 20.22 & - & - \\
\hline
\end{tabular}

$A C L$ anterior cruciate ligament, $D M C L$ deep medial collateral ligament, $P C L$ posterior cruciate ligament, $S M C L$ superficial medial collateral ligament

\section{Discussion}

Establishment of the knee joint model

The knee joint is one of the most complex joints in the human body, with a complex anatomic structure and biomechanical properties. The traditional mechanical method to study its biomechanical functions usually involves the application of extra-articular loads and use of mechanical measuring instruments [9], and it is difficult to investigate the stress distribution within the joints and other issues using this method. Therefore, establishing knee joint models such as the crossed fourlink physical model and the two-dimensional mathematical model of the sagittal knee joint as well as the three-dimensional model of dynamic response of the knee joint has become an important measure for further studying the biomechanical characteristics of knee joints [10]. Since its first application in orthopedic biomechanics by Brekelmans et al. [11] in 1972, the FEM has been widely used in modeling teeth, artificial limbs, 

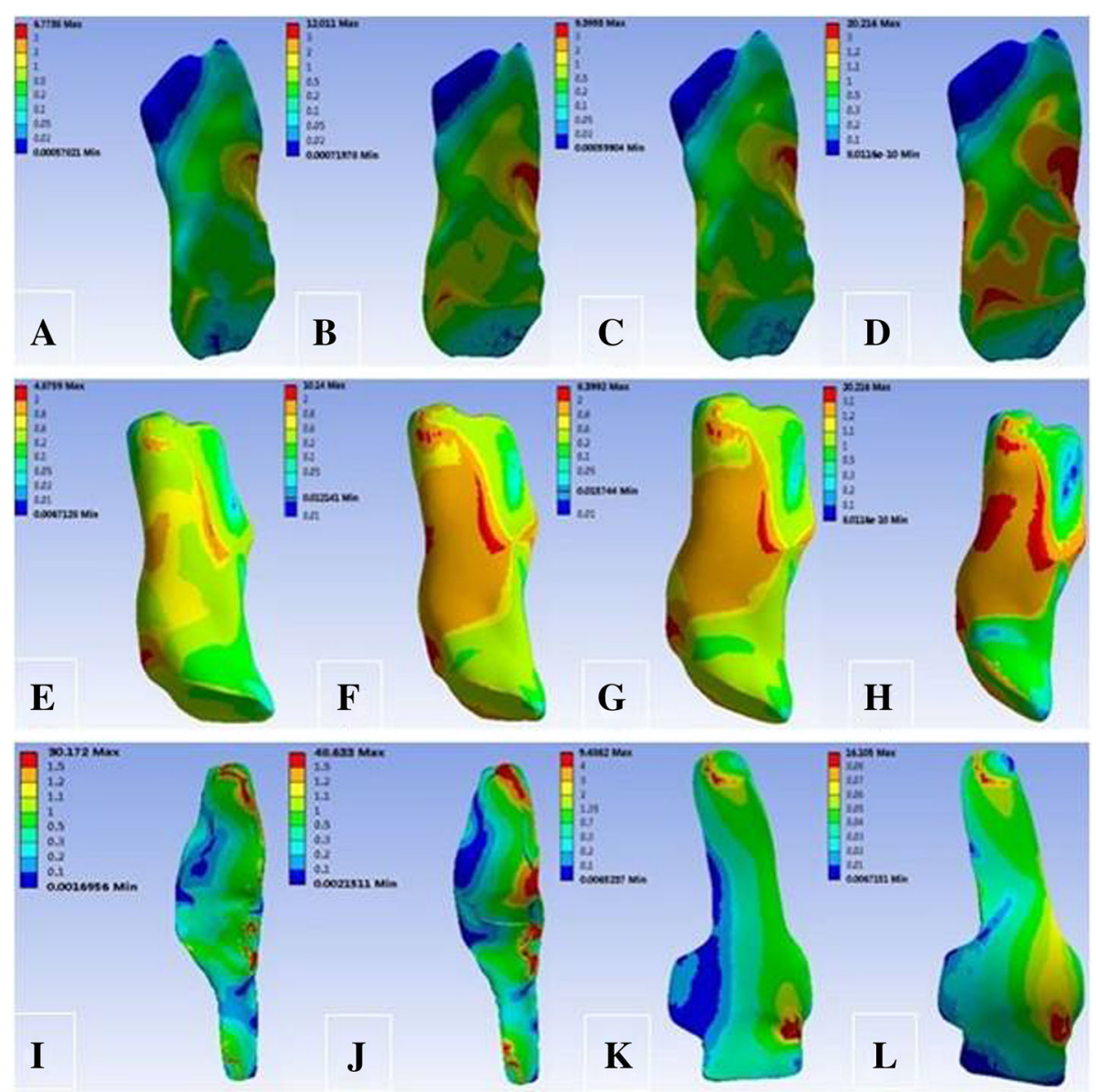

Fig. 5 von Mises stress distribution of $A C L, P C L, S M C L$, and $D M C L$ under $10 \mathrm{~N}$ m of valgus moment. a $A C L$ in case 1. b $A C L$ in case 2. c $A C L$ in case 3. $\mathbf{d}$ ACL in case 4. e PCL in case 1. $\mathbf{f} P C L$ in case 2. $\mathbf{g} P C L$ in case 3. $\mathbf{h} P C L$ in case 4. $\mathbf{i} S M C L$ in case 1. $\mathbf{j} S M C L$ in case 3. $\mathbf{k} D M C L$ in case 1. I DMCL in case 2

spine, etc. [12] and has been gradually applied to the biomechanics of ankles, knees, wrists, and other joints $[13,14]$.

Single-mode CT or MR images are typically unable to provide a clear contrast for intact knee joints, leading to difficulty in accurately constructing an FEM of knee joints containing multiple anatomic structures. Studies have found that although CT

Table 5 Response parameters of the knee joint under $10 \mathrm{~N} \mathrm{~m}$ of external rotation moment

\begin{tabular}{llllll}
\hline & $\begin{array}{l}\text { Tibial external } \\
\text { rotation angle }\left(^{\circ}\right)\end{array}$ & \multicolumn{4}{l}{ Peak stress (MPa) } \\
\cline { 3 - 6 } & & $\mathrm{ACL}$ & $\mathrm{PCL}$ & $\mathrm{SMCL}$ & $\mathrm{DMCL}$ \\
\hline Case 1 & 5.92 & 8.45 & 6.79 & 13.76 & 4.39 \\
Case 2 & 5.95 & 9.66 & 7.67 & - & 4.45 \\
Case 3 & 5.94 & 10.69 & 8.96 & 16.08 & - \\
Case 4 & 6.10 & 11.35 & 9.67 & - & - \\
\hline
\end{tabular}

$A C L$ anterior cruciate ligament, $D M C L$ deep medial collateral ligament, $P C L$ posterior cruciate ligament, $S M C L$ superficial medial collateral ligament image data alone can be used to accurately construct bone structure models, it cannot be used to accurately simulate the cartilage, ligament, meniscus, and other soft tissues [15]. In contrast, MR imaging data alone can be used to accurately construct the anatomic structure models of various soft tissues including knee joints, while it cannot be used to accurately simulate bone structures [16]. Thus, using CT or MR imaging alone will significantly decrease the accuracy of these models, leading to inaccurate mechanical analysis of the knee joints.

Yao et al. [17] accurately constructed FEMs of the femoral cartilage, tibial cartilage, and medial meniscus using MATLAB and Hypermesh software, but not of other structures of knee joints. Therefore, a single software often has some limitations, and the constructed FEM fails to truly represent the anatomic characteristics of knee joints; thus, the FEMs of knee joints can be accurately constructed only by collaborative application of a variety of modeling software. 

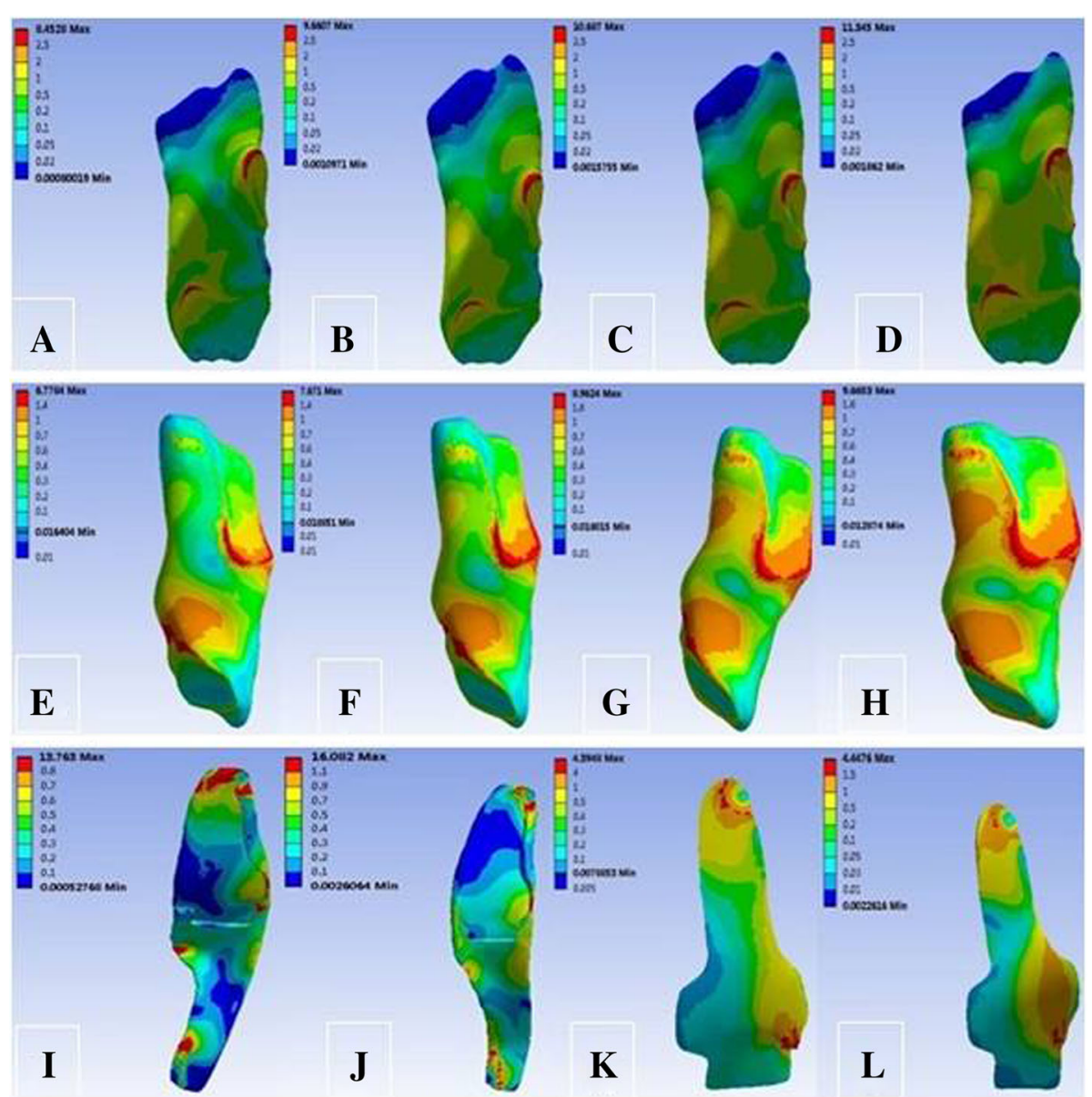

Fig. 6 von Mises stress distribution of the $A C L, P C L, S M C L$, and $D M C L$ under $10 \mathrm{~N}$ m of external rotation moment. a $A C L$ in case 1 . b $A C L$ in case 2. $\mathbf{c ~ A C L}$ in case 3. $\mathbf{d}$ ACL in case 4. e PCL in case 1. $\mathbf{f} P C L$ in case 2. $\mathbf{g} P C L$ in case 3. $\mathbf{h} P C L$ in case 4. i SMCL in case 1. j SMCL in Case 3. $\mathbf{k} D M C L$ in case 1. I DMCL in case 2

In this study, a variety of modes of CT and MR imaging as well as MIMICS 14.0, Geomagic Studio 12.0, and ANSYS modeling software were applied to construct a three-dimensional FEM of knee joints using the reverse engineering (RE) principle. In the MIMICS software, the structures in $\mathrm{CT}$ and $\mathrm{MR}$ images were assembled according to the human anatomy. However,

Table 6 Response parameters of the knee joint under $10 \mathrm{~N} \mathrm{~m}$ of internal rotation moment

\begin{tabular}{llllll}
\hline & $\begin{array}{l}\text { Tibial internal } \\
\text { rotation angle }\left(^{\circ}\right)\end{array}$ & \multicolumn{4}{l}{ Peak stress (MPa) } \\
\cline { 3 - 6 } & & $\mathrm{ACL}$ & $\mathrm{PCL}$ & $\mathrm{SMCL}$ & $\mathrm{DMCL}$ \\
\hline Case 1 & 6.64 & 9.06 & 7.26 & 14.75 & 4.71 \\
Case 2 & 7.48 & 10.35 & 8.22 & - & 4.77 \\
Case 3 & 6.72 & 11.45 & 9.60 & 17.23 & - \\
Case 4 & 7.57 & 12.16 & 10.36 & - & - \\
\hline
\end{tabular}

$A C L$ anterior cruciate ligament, $D M C L$ deep medial collateral ligament, $P C L$ posterior cruciate ligament, $S M C L$ superficial medial collateral ligament this assembled model was very coarse due to the presence of interference surfaces. This issue could be addressed using Geomagic Studio 12.0, in which the interference surfaces in the model were removed. However, this software was not able to generate ANSYS preprocessing files; therefore, the repaired models were imported again into the 3-matic module of MIMICS software for initial meshing before being imported into ANSYS for finite element analysis (FEA). In addition, since this study focused on the mechanical analyses of the ligaments, their mesh size was refined at $1 \mathrm{~mm}$, and the bone structures were set as a solid body with a mesh size of $4 \mathrm{~mm}$, reflecting the different focuses of subjects in FEA. Because the meshing quality determined the accuracy of the FEA results, an even finer mesh would be required to analyze non-linear contacts. Local meshes with poor quality were optimized, and interactive meshes with satisfactory 6-node pentahedrons and 8node hexahedrons were obtained based on the high- 

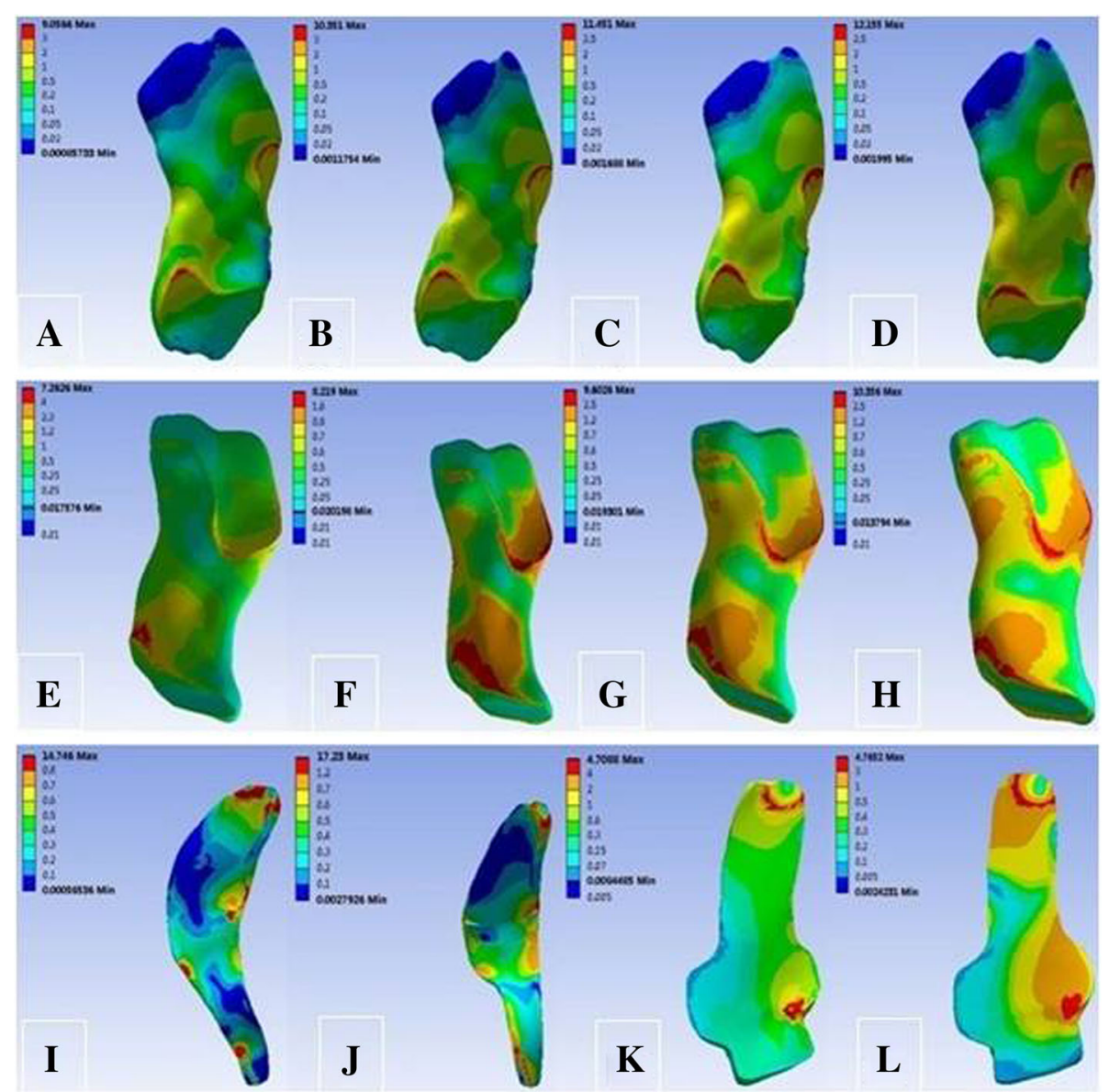

Fig. 7 von Mises stress distribution of the $A C L, P C L, S M C L$, and $D M C L$ under $10 \mathrm{~N} \mathrm{~m}$ of internal rotation moment. a $A C L$ in $c a s e 1$. b $A C L$ in case 2. $\mathbf{c ~ A C L}$ in case 3. $\mathbf{d}$ ACL in case 4. e PCL in case 1. $\mathbf{f} P C L$ in case 2. $\mathbf{g} P C L$ in case 3. $\mathbf{h} P C L$ in case 4. i SMCL in case 1. $\mathbf{j}$ SMCL in case 3. k DMCL in case 1. I DMCL in case 2

quality area meshes, in order to achieve more accurate results compared to those obtained using meshes with 10-node tetrahedron elements. Ultimately, a three-dimensional model of the human right knee joint containing a variety of anatomic structures, such as the middle and upper segments of the femur, middle and upper segments of the tibia, fibula, patella, meniscus, ACL, PCL, MCL, lateral collateral ligament, and patellar ligament, was constructed. Meanwhile, high-quality volume meshes were developed, satisfying the requirements for FEA of biomechanics of knee joints. This FEM can be used to analyze the stress distribution of ligaments, contacts of tibiofemoral joints, stress distribution on articular surface, changes of stress distribution under different ligament deficiencies, and other biomechanical studies, as well as to simulate the effects of surgical results on the biomechanics of knee joints under different surgical conditions, and conduct biomechanical analyses of surgical fixations.

\section{Biomechanical analyses of medial collateral ligament of} the knee joint

The anatomy of MCL has been extensively studied [5]. In this experiment, based on the human anatomy, an FEM of the knee joint was established to simulate the anterior-posterior translation, valgus-varus rotation, and internal-external rotation of the knee joint, so as to study the biomechanical functions of its superficial and deep MCLs, in which the knee joint varus was excluded because the knee MCL is completely relaxed in this condition. In the experiment, a gradually increasing color grading from blue to red color indicated gradually increasing von Mises stress, which represented a greater load on the ligament and a greater role of the site and likelihood of damage.

Under the load of the 134- $\mathrm{N}$ forward force, the tibial displacement changed from $4.89 \mathrm{~mm}$ at intact MCL to 5.17, 5.04, and $5.17 \mathrm{~mm}$ at SMCL deficiency, DMCL deficiency, and overall MCL deficiency, respectively. A greater variation of tibial displacement at overall MCL 
deficiency indicated that MCL plays a role in limiting the forward translation of the tibia. Meanwhile, the tibial displacement showed a greater variation at SMCL deficiency compared with that at DMCL deficiency, suggesting that SMCL has a greater effect than the DMCL. During this process, the stress at ACL maintained a maximum value, suggesting that ACL plays the most important role in limiting the tibial anterior translation. Moreover, a greater stress at SMCL than that at DMCL indicated that the SMCL has a greater effect. The stress nephogram showed that the peak stresses at ACL and SMCL were mainly located at the femoral end point, indicating that during tibial anterior translation, injury to the femoral end point is most likely to occur at ACL and SMCL, and less likely to occur at DMCL.

Under the load of the 134-N backward force, the tibial translation showed a very small variation with MCL deficiency, during which the stress at PCL maintained a maximum value, while the stresses at SMCL and DMCL were relatively small, suggesting that PCL plays the most important role in constraining the tibial posterior translation, while the effects of SMCL and DMCL are very small. Meanwhile, the peak stress at PCL occurred at the tibial start and end points, suggesting that in tibial posterior translation, injury is most likely to occur at the femoral start and end points at PCL, while the risk of injury at SMCL and DMCL is small.

Under the load of the $10-\mathrm{N} \mathrm{m}$ valgus torque, the tibial valgus angle showed a large variation with MCL deficiency, which changed from $4.06^{\circ}$ at intact MCL to $6.08^{\circ}, 4.86^{\circ}$, and $6.22^{\circ}$ at SMCL deficiency, MCL deficiency, and overall MCL deficiency, respectively, suggesting that MCL tends to resist the valgus motion of knee joints. Meanwhile, the stress was the largest at SMCL followed by that at DMCL, indicating that SMCL plays the most important role in limiting the valgus motion and the effect of DMCL is relatively smaller. As evident in the stress nephogram, the peak stress at SMCL occurred at the end point and anterior part of the femur, indicating that injury is most likely to occur at the end point and anterior part of the femur in valgus motion of knee joints at SMCL. In contrast, the peak stress at DMCL occurred at the femoral start and end points, suggesting that they are prone to injury at DMCL.

Under the load of the $10-\mathrm{N} \mathrm{m}$ external rotation torque, the tibial external rotation angle changed from $5.92^{\circ}$ at intact $\mathrm{MCL}$ to $5.95^{\circ}, 5.94^{\circ}$, and $6.10^{\circ}$ at SMCL deficiency, DMCL deficiency, and overall MCL deficiency, respectively. The tibial external rotation angle showed a large variation at overall MCL deficiency, suggesting that MCL tends to resist the external rotation of the knee joints. Although the tibial external rotation angle did not show significant difference between SMCL and DMCL deficiencies, the stress at SMCL was larger than that at DMCL, indicating that SMCL plays a more significant role in limiting the external rotation of the knee joint than the DMCL. As observed in the stress nephogram, the peak stress at SMCL was mainly located at the femoral end point and posterior part, indicating that they are prone to injury at SMCL during external rotation of knee joints, while the injury at DMCL was smaller.

Under the load of the 10- $\mathrm{N} \mathrm{m}$ internal rotation torque, the tibial internal rotation angle changed from $6.64^{\circ}$ at intact MCL to $7.48^{\circ}, 6.72^{\circ}$, and $7.57^{\circ}$ at SMCL deficiency, DMCL deficiency, and overall MCL deficiency, respectively. The tibial internal rotation angle showed a larger variation than the tibial external rotation angle with MCL deficiency, suggesting that knee joint MCL has a greater effect on limiting the internal rotation than the external rotation. Similarly, greater stress at SMCL than that at DMCL indicated that the SMCL has a greater effect on limiting the internal rotation of knee joints than the DMCL. As observed in the stress nephogram, the peak stress at SMCL occurred at the femoral end point, indicating that the femoral end point was prone to injury at SMCL during internal rotation of knee joints, while the injury at DMCL was smaller.

The above analyses show that in the extended position of knee joints, the main effect of MCL is to resist the valgus motion of knee joints, along with limiting the tibial forward displacement as well as the internal and external rotations of knee joints. The SMCL plays the most important role in the structure of the MCL of knee joints, while the effects of DMCL are relatively lesser. In various motions of knee joints, the femoral end point at SMCL is the most prone to injury. The anterior part of the femur is more prone to injury in resisting valgus motion, and the posterior part in resisting external rotation at SMCL. However, injury is less likely to occur at DMCL, and when it does occur, it occurs at the femoral start and end points.

\section{Conclusions}

In summary, this model to evaluate the function of the MCL by FEA is reliable [18-20]. The results indicate that the knee MCL II injury should be repaired by surgery. However, the shortcoming of this study is the lack of clinical evidence. We hope to achieve a further investigation in the clinic.

\footnotetext{
Abbreviations

ACL: Anterior cruciate ligament; CT: Computed tomography; DICOM: Digital Imaging and Communications in Medicine; DMCL: Deep medial collateral ligament; E: Young's modulus; FEA: Finite element analysis; FEM: Finite element model; LCL: Lateral collateral ligament; MCL: Medial collateral ligament; MIMICS: Materialise Interactive Medical Image Control System; MRI: Magnetic resonance imaging; PCL: Posterior cruciate ligament; PL: Patellar ligament; RE: Reverse engineering; SMCL: Superficial medial collateral ligament; $V$ : Poisson's ratio
} 


\section{Acknowledgements}

We thank MedSci who provided medical writing services.

\section{Funding}

No authors received any funding.

\section{Availability of data and materials}

Please contact the author for data requests.

\section{Authors' contributions}

DR contributed to this article by making substantial contributions to the conception and design of the study. XZ and DR contributed equally to this paper. $Y L$ contributed to the acquisition, analysis, and interpretation of the data. PW performed the operations. ZS, and JL were involved in drafting the manuscript. All authors read and approved the final manuscript.

\section{Competing interests}

The authors declare that they have no competing interests.

\section{Consent for publication}

Informed consent for the publication of details, images, or videos relating to the individual participant was obtained from the participant.

\section{Ethics approval and consent to participate}

This research has been approved by the ethics committee of the Third Hospital of Hebei Medical University. And the reference number is 2016-005-1. Informed consent to participate in the study was obtained from the participant.

\section{Publisher's Note}

Springer Nature remains neutral with regard to jurisdictional claims in published maps and institutional affiliations.

\section{Author details}

${ }^{1}$ Third Hospital of Hebei Medical University, Shijiazhuang 050051, China. ${ }^{2}$ Hebei Provincial Key Laboratory of Orthopaedic Biomechanics, Shijiazhuang 050051, Hebei, China. ${ }^{3}$ Department of Orthopedic Center, Third Hospital of Hebei Medical University, 139 Zi Qiang Road, Shijiazhuang 050051, Hebei, China.

Received: 26 February 2017 Accepted: 12 April 2017

Published online: 21 April 2017

\section{References}

1. Hetsroni I, Mann G. Combined reconstruction of the medial collateral ligament and anterior cruciate ligament using ipsilateral quadriceps tendonbone and bone-patellar tendon-bone autografts. Arthrosc Tech. 2016;5(3): e579-87.

2. Najibi S, Albright JP. The use of knee braces, part 1: prophylactic knee braces in contact sports. Am J Sports Med. 2005;33(4):602-11.

3. Hamilton TW, Strickland LH, Pandit HG. A meta-analysis on the use of gabapentinoids for the treatment of acute postoperative pain following total knee arthroplasty. J Bone Joint Surg Am. 2016;98(16):1340-50.

4. Yenchak AJ, et al. Criteria-based management of an acute multistructure knee injury in a professional football player: a case report. J Orthop Sports Phys Ther. 2011;41(9):675-86.

5. Liu F, et al. Morphology of the medial collateral ligament of the knee. J Orthop Surg Res. 2010;5(1):69

6. Pena $E$, et al. A finite element simulation of the effect of graft stiffness and graft tensioning in ACL reconstruction. Clin Biomech (Bristol, Avon). 2005; 20(6):636-44.

7. Shirazi R, Shirazi-Adl A. Computational biomechanics of articular cartilage of human knee joint: effect of osteochondral defects. J Biomech. 2009:42(15): 2458-65.

8. Gabriel MT, et al. Distribution of in situ forces in the anterior cruciate ligament in response to rotatory loads. J Orthop Res. 2004;22(1):85-9.

9. Hinterwimmer S, Baumgart R, Plitz W. Tension changes in the collateral ligaments of a cruciate ligament-deficient knee joint: an experimental biomechanical study. Arch Orthop Trauma Surg. 2002;122(8):454-8.

10. Abdel-Rahman EM, Hefzy MS. Three-dimensional dynamic behaviour of the human knee joint under impact loading. Med Eng Phys. 1998;20(4):276-90.
11. Brekelmans WA, Poort HW, Slooff TJ. A new method to analyse the mechanical behaviour of skeletal parts. Acta Orthop Scand. 1972:43(5):301-17.

12. Silva MJ, Keaveny TM, Hayes WC. Load sharing between the shell and centrum in the lumbar vertebral body. Spine (Phila Pa 1976). 1997;22(2): 140-50.

13. Li G, et al. A validated three-dimensional computational model of a human knee joint. J Biomech Eng. 1999;121(6):657-62.

14. LeRoux MA, Setton LA. Experimental and biphasic FEM determinations of the material properties and hydraulic permeability of the meniscus in tension. J Biomech Eng. 2002;124(3):315-21.

15. Chantarapanich $\mathrm{N}$, et al. A finite element study of stress distributions in normal and osteoarthritic knee joints. J Med Assoc Thai. 2009;92 Suppl 6: S97-103.

16. Donahue $\mathrm{TL}$, et al. A finite element model of the human knee joint for the study of tibio-femoral contact. J Biomech Eng. 2002;124(3):273-80.

17. Yao J, et al. Stresses and strains in the medial meniscus of an ACL deficient knee under anterior loading: a finite element analysis with image-based experimental validation. J Biomech Eng. 2006;128(1):135-41.

18. Liu F, et al. In vivo length patterns of the medial collateral ligament during the stance phase of gait. Knee Surg Sports Traumatol Arthrosc. 2011;19(5): 719-27.

19. Ellis BJ, et al. Medial collateral ligament insertion site and contact forces in the ACL-deficient knee. J Orthop Res. 2006;24(4):800-10.

20. Hosseini $A$, et al. In vivo length change patterns of the medial and lateral collateral ligaments along the flexion path of the knee. Knee Surg Sports Traumatol Arthrosc. 2015;23(10):3055-61.

\section{Submit your next manuscript to BioMed Central and we will help you at every step:}

- We accept pre-submission inquiries

- Our selector tool helps you to find the most relevant journal

- We provide round the clock customer support

- Convenient online submission

- Thorough peer review

- Inclusion in PubMed and all major indexing services

- Maximum visibility for your research

Submit your manuscript at www.biomedcentral.com/submit 